\title{
ON THE REFLECTION OF ALFVÉN WAVES IN AN IDEAL MAGNETOATMOSPHERE
}

\author{
HADI YAHYA ALKAHBY \\ Department of Mathematics \\ Dillard University \\ New Orleans, LA 70122, U.S.A. \\ and
}

M.A. MAHROUS

Department of Mathematics, Universty of New Orleans, New Orleas, LA , 70148, U.S.A.

(Received March 20, 1996)

\begin{abstract}
A linearized theory of magnetoatmospheric waves is developed where the restoring forces are those of compressibility and magnetic pressure. An equation for resonance is derived. Reflection and tunneling of upward propagating Alfvén waves in an ideal Magnetoatmosphere are considered. It is shown that the magnetic field produces a reflecting nonabsorbing critical layer. Below the critical layer, the solution of the problem can be written as a linear combination of an upward and a downward propagating wave and above it the solution decays exponentially with the altitude. The location of the critical layer and the magnitude of the reflection coefficient are determined and the conclusions are discussed in connection with the heating mechanism of the solar atmosphere.
\end{abstract}

KEY WORDS: Magnetoatmosphere, critical layer, wave propagation

1991 AMS SUBJECT CLASSIFICATION CODES: 76N,76Q

\section{INTRODUCTION}

The dynamics of the solar atmosphere is complicated by the fact that not only is it strongly stratified, in both gas density and temperature, but it is also permeated by a non-uniform magnetic field. The solar atmosphere is an example of a plasma that is both structured and stratified. More specifically, the sun is a compressible plasma and able to support sound waves. The presence of a strong magnetic field indicates that the solar atmosphere is an elastic medium. Thus, wave motions of various types will occur and become a source of energy in the solar atmosphere.

In this paper a linearized theory of magnetoatmospheric waves, involving the combined restoring forces due to compressibility and magnetic pressure, is developed for the case of a uniform horizontal magnetic field. A general propagation equation is derived for adiabatic perturbations with arbitrary vertical distribution of Alfven and sound speeds. An exact analytical solution of the propagation equation is obtained for the case of an isothermal atmosphere permeated by a uniform horizontal magnetic field. We examine the propagation of Alfven waves in two distinct regions in the solar atmosphere, which is taken to be an ideal one. It is shown that in the first region, where the strength of the magnetic field is weak, the solution can be written as a linear combination of upward and downward propagating waves 
with equal wavelengths. In the first region the motion is approximately acoustic because the motion is dominated by the restoring force of compressibility. In the second region, where the magnetic field strength dominates the motion, the solution decays exponentially with altitude. The first and second regions are connected by a critical layer in which the reflection takes place and the motion is influenced greatly by the effect of the magnetic field. Also, the behavior of the solution, in both regions, indicates that the tunneling is weak as the waves, of all kinds, propagate between the two regions and the reflection is very strong. We expect the motion, in both regions to continue in its prescribed form because there is no physical mechanism for dissipation. The reflection coefficient, location of the transition region, critical layer, and the conclusions are presented in connection with the heating mechanism of the solar atmosphere.

Finally, in the formulation of the problem we will be able to introduce and justify the so-called "magnetic energy condition" in an ideal Magnetoatmosphere as an upper boundary condition to ensure a unique solution.

\section{PROBLEM FORMULATION}

We consider an ideal magnetoatmosphere which is inviscid and thermally non-conducting, and occupies the upper half-space $z>0$. It is assumed that the gas is under the influence of a uniform horizontal magnetic field and that it has an infinite electrical conductivity. We investigate the problem of small vertical oscillations about equilibrium.

Let the equilibrium pressure, density, magnetic field intensity and external potential be denoted by $\mathrm{P}_{0}(\mathrm{z}), \rho_{0}(\mathrm{z}), \mathrm{B}(\mathrm{z})$ and $\psi_{0}(\mathrm{z})$. Let $\mathrm{P}, \rho, \mathrm{B}, \psi$ and $\mathbf{V}$ be the perturbations in the pressure, density, magnetic field intensity, external potential and velocity. The equations of ideal magnetohydrodynamics are those of momentum, induction, isotropy, and continuity:

$$
\begin{gathered}
\rho\left[\frac{\partial \mathbf{V}}{\partial t}+\mathbf{V} \cdot \nabla \mathbf{V}\right]=-\nabla p-\mathbf{B} \times(\nabla \times \mathbf{B})-\rho \nabla \psi, \\
\frac{\partial \mathbf{B}}{\partial t}=\nabla \times(\mathbf{V} \times \mathbf{B}) \\
{\left[\frac{\partial}{\partial t}+\mathbf{V} \cdot \boldsymbol{\nabla}\right]\left(P \rho^{-\gamma}\right)=0} \\
{\left[\frac{\partial}{\partial t}+\mathbf{V} \cdot \boldsymbol{\nabla}\right] \rho=-\rho \nabla \cdot \mathbf{V}}
\end{gathered}
$$

Here $\gamma$ denotes the ratio of the specific heats. These equations are linearized about a static equilibrium defined by

$$
\boldsymbol{\nabla} P_{0}+\mathbf{B}_{0} \times\left(\nabla \times \mathbf{B}_{0}\right)=-\rho_{0} \nabla \psi_{0}
$$

In this problem $\psi_{0}$ is considered to represent a uniform gravitational field. Thus, $\nabla \psi_{0}=-\mathbf{g}=$ $(0,0, g)$ and the last term in equations (2.1) and (2.5) is replaced by $\rho_{0} g$. In terms of the linear Lagrangian displacement field $\xi(r, t)$, the velocity perturbation is defined by:

$$
\mathbf{V}=\frac{\partial \boldsymbol{\xi}}{\partial t}
$$

and the equation of motion of ideal magnetohydrodynamic is obtained by integrating and eliminating all perturbation quantities except $\xi$. As a result, we have

$$
\rho_{0} \frac{\partial^{2} \boldsymbol{\xi}}{\partial t^{2}}=\mathbf{F}(\boldsymbol{\xi})
$$


where the force operator $F(\xi)$ is defined by

$$
\mathbf{F}(\boldsymbol{\xi})=\boldsymbol{\nabla}\left(\gamma P_{0} \boldsymbol{\nabla} \cdot \boldsymbol{\xi}+\boldsymbol{\xi} \cdot \boldsymbol{\nabla} P_{0}\right)-\mathbf{B}_{0} \times(\boldsymbol{\nabla} \times \mathbf{R})-\mathbf{R} \times\left(\boldsymbol{\nabla} \times \mathbf{B}_{0}\right)+\boldsymbol{\nabla} \cdot\left(\rho_{0} \boldsymbol{\xi}\right) \boldsymbol{\nabla} \psi_{0},
$$

where

$$
\mathbf{R}=\boldsymbol{\nabla} \times\left(\boldsymbol{\xi} \times \mathbf{B}_{0}\right)
$$

The linear Lagrangian displacement field is assumed to be of the form $\xi(x, y, z, t)$ and as an integral superposition of harmonic terms, with $\xi$ and its first $x$ derivative vanishing at infinity. As a result,

$$
\xi=\frac{1}{2 \pi} \int_{-\infty}^{\infty} e^{-\imath \omega t} \phi(z, k) e^{\imath k x} d k
$$

where the wave number is assumed to be $\mathbf{k}=(k, 0,0)$. Consequently, the horizontal magnetic field can be written as

$$
\mathbf{B}_{0}=\left(B_{0}(z), B_{y}(z), 0\right) .
$$

Using equation (2.10), equation (2.7) becomes

$$
-\rho_{0} \omega^{2} \phi=\mathbf{F}(\phi, k) .
$$

Eliminating all variables except $\phi_{z}$, we obtain the following differential equation:

$$
\frac{d}{d z}\left[A(z, \omega) \frac{d \phi_{z}}{d z}\right]+C(z, \omega) \phi=0
$$

where the coefficient $A(z, \omega)$ and $C(z, \omega)$ have the following form:

$$
\begin{gathered}
A(z, \omega)=\frac{\rho_{0} A_{11} A_{12} A_{13}}{A_{14} A_{15}} \\
C(z, \omega)=\rho_{0}\left[A_{12}-\frac{k^{2} g^{2} A_{12}}{A_{14} A_{15}}-\frac{k^{2} g}{\rho_{0}}\left(\frac{d}{d z}\left[\rho_{0}\left(\frac{A_{11}-a_{x}}{A_{14} A_{15}}\right) A_{16}\right]\right)\right],
\end{gathered}
$$

where

$$
\begin{aligned}
& A_{11}=a_{x}^{2}+a_{y}^{2}+c_{0}^{2}, \quad A_{12}=\omega^{2}-a_{x}^{2} k^{2}, \\
& A_{13}=\omega^{2}-\frac{a_{x}^{2} k^{2} c_{0}^{2}}{A_{11}-a_{y}}, \quad A_{14}=\omega^{2}-\frac{1}{2}\left[k^{2} A_{11}-A_{17}\right], \\
& A_{15}=\omega-\frac{1}{2}\left[k^{2} A_{11}+A_{17}\right], \quad A_{16}=\omega-\left[\frac{a_{x} c_{0}^{2} k^{2}}{A_{11}-a_{x}}\right], \\
& A_{17}=k^{4}\left[A_{11}^{2}-4 a_{x}^{2} c_{0}^{2}\right] .
\end{aligned}
$$

The parameters $a_{x}$ and $a_{y}$ are the $\mathrm{x}$ and $\mathrm{y}$ components of Alfven speed, while $c_{0}^{2}$ denotes the speed of sound.

\section{SIMPLIFICATION OF THE PROBLEM AND BOUNDARY CONDITIONS}

For this problem the atmosphere is assumed to be isothermal $\left(c_{0}=c(z)=\right.$ constant) and permeated by a uniform horizontal magnetic field $\mathrm{B}=(B, 0,0)$. The equilibrium pressure $P_{0}$ and constant temperature $T_{0}$ satisfy the gas law $P_{0}=R T_{0} \rho_{0}$ and the hydrostatic equation $P_{0}^{\prime}(z)+g \rho_{0}=0$. Here, $\mathrm{R}$ denotes the gas constant and the prime denotes differentiation with respect with respect to $z$. As a result, the equilibrium pressure and density can be written as:

$$
P_{0}(z)=P_{0}(0) \exp (-z / H), \quad \rho_{0}(z)=\rho_{0}(0) \exp (-z / H),
$$


where $H=-\rho_{0}^{\prime}(z) / \rho_{0}(z)=\frac{R T_{0}}{g}$ is the density scale height. Consequently, the differential equation (2.13) becomes

$$
\begin{gathered}
{\left[\omega^{2} c^{2}+\left(\omega^{2}-c^{2} k^{2}\right) \alpha^{2}(0) \exp \left(\frac{z}{H}\right)\right] \phi^{\prime \prime}(z)-\frac{c^{2} \omega^{2}}{H} \phi^{\prime}(z)+} \\
{\left[\left(\omega^{2}-c^{2} k^{2}\right)\left(\omega^{2}-k^{2} \alpha_{0}^{2}(0) \exp \left(\frac{z}{H}\right)-g\left(g-\frac{c^{2}}{H}\right) k^{2}\right] \phi(z)=0,\right.}
\end{gathered}
$$

where

$$
\alpha_{0}^{2}(0)=\frac{B^{2}}{4 \pi \rho_{0}(0)}
$$

denotes Alfven speed at $z=0$. Furthermore, we introduce the following dimensionless quantities and variables:

$$
\begin{gathered}
\phi(z)=W(x) \exp -k z, \quad x=x_{0} \exp \left(-\frac{z}{H}\right), \\
\dot{z}=\frac{z}{H}, \quad k=H k, \quad x_{0}=\frac{\beta^{2} \sigma^{2}}{k^{2}-\sigma^{2}}, \quad \beta^{2}=\frac{\alpha_{0}^{2}(0)}{c^{2}}, \quad \sigma=\frac{H \omega}{c},
\end{gathered}
$$

where the prime on $\mathrm{z}$ is eliminated for simplicity. Thus, the differential equation (3.2) becomes,

$$
x(1-x) \frac{d^{2} W(x)}{d x^{2}}+[c-(a+b+1) x] \frac{d W(x)}{d x}-a b W(x)=0 .
$$

It is clear that the differential equation (3.5) is a special case of the hypergeometric differential equation with

\section{Boundary Conditions:}

$$
a+b=c=2 k+1, \quad a b=\sigma^{2}+k+\left(\frac{\gamma-1}{\gamma}\right) \frac{k^{2}}{\sigma^{2}},
$$

To complete the formulation of the problem, certain conditions must be imposed to ensure a unique solution. If the atmosphere is viscous, an appropriate condition would be the dissipation condition, which requires the finiteness of the rate of the energy dissipation in an infinite column of fluid of unit cross-section. Since the dissipation function depends on the squares of the velocity gradients, this implies

$$
\int_{0}^{\infty}\left|W^{\prime}\right|^{2} d x<\infty
$$

In our problem the atmosphere is not viscous, but the integral in (3.7) is proportional to the magnetic energy in an infinite column of fluid. This condition is a reasonable one to apply so long as there is no energy radiation to infinity, which is true in our case, and we shall call this condition "magnetic energy condition." Thus we will require (3.7) even when the atmosphere is inviscid. A boundary condition is also required at $\mathrm{x}=0$, and we shall set

$$
W(0)=1,
$$

by suitably normalizing $\mathrm{W}(\mathrm{x})$. It will be seen that the boundary conditions (3.7) and (3.8) will determine a unique solution to within a multiplicative constant.

\section{SOLUTION OF THE PROBLEM}

In this section we investigate solutions of the following differential equation,

$$
x(1-x) \frac{d^{2} W(x)}{d x^{2}}+[c-(a+b+1) x] \frac{d W(x)}{d x}-a b W(x)=0,
$$

where

$$
a+b=c=2 k+1, \quad a b=\sigma^{2}+k+\left(\frac{\gamma-1}{\gamma}\right) \frac{k^{2}}{\sigma^{2}}
$$


subject to the prescribed boundary conditions. Solving for the parameters a and $b$, we obtain

$$
a=\frac{1}{2}+k-r, \quad b=\frac{1}{2}+k+r
$$

where

$$
r=\sqrt{r_{1}-r_{2}}, \quad r_{1}=\frac{1}{4}+k^{2} \quad \text { and } \quad r_{2}=\sigma^{2}+\frac{\gamma-1}{\gamma} \frac{k^{2}}{\sigma^{2}} .
$$

It is clear that the parameter $\mathrm{r}$ is a real number, for $\mathrm{r}_{1}>\mathrm{r}_{2}, \mathrm{r}=0$ for $\mathrm{r}_{1}=\mathrm{r}_{2}$ and $r=i \sqrt{r_{2}-r_{1}}$ for $r_{1}<r_{2}$. The differential equation (4.1) is a special case of the hypergeometric equation which has three regular singular points at $\mathrm{x}=0, \mathrm{x}=1$ and $\mathrm{x}=\infty$. The intermediate regular singular point, $\mathrm{x}=1$, corresponds to the existence of the critical layer, which has a great importance for understanding the heating mechanism of the solar atmosphere. As a result, the differential equation (4.1) has two linearly independent solutions which can be written in the following form for $|x|<1$.

$$
\begin{gathered}
W_{1}(x)=F(a, b ; c ; x), \\
W_{2}(x)=x^{1-c} F(a-c+1, b-c+1 ; 2-c ; x), \\
F(a, b ; c ; x)=\frac{\Gamma(c)}{\Gamma(a) \Gamma(b)} \sum_{n=0}^{\infty} \frac{\Gamma(a+n) \Gamma(b+n)}{\Gamma(c+n)} \frac{x^{n}}{n !} .
\end{gathered}
$$

Since $k>0$, then $1-c=-2 k<0$, and

$$
x^{1-c}=\left[x_{0} \exp (-z)\right]^{1-c} \rightarrow \infty \text {, as } z \rightarrow \infty .
$$

Thus, the solution of the differential equation (4.1) as defined by equation (4.5) will be eliminated by the magnetic energy condition. Consequently, the general solution of the differential equation (4.1) is

$$
W_{1}(x)=A F(a, b ; c ; x),
$$

where $\mathrm{A}$ is an arbitrary constant and can be determined by the boundary condition (3.8). Using the asymptotic behavior of $F(a, b ; c ; x)$ for $|x|>1$ and reintroducing the variable $z$, the solution of (4.4) can be written as

$$
W(z)=\text { Cons } \cdot\left[\left[\exp \left(\frac{1}{2}+k+i r\right) z\right]+R \exp \left(\frac{1}{2}+k-i r\right) z\right],
$$

where $\mathrm{R}$ denotes the reflection coefficient and defined by

$$
R=\exp \left[i\left(\theta+2 r \log x_{0}\right)\right]
$$

and

$$
\theta=\arg (R)=\arg \left[\frac{\Gamma(a) \Gamma(b-a)}{\Gamma(a-b) \Gamma(b)} \frac{\Gamma(c-b)}{\Gamma(c-a)}\right]
$$

\section{GENERAL DISCUSSION}

The structured nature of the solar magnetic field means that magnetism is of greater importance in some regions of the sun than in others. A simple guide to the relative importance of magnetic effects is provided by the plasma beta, $\beta$, defined by

$$
\beta=\frac{P_{0}}{P_{m}}=\frac{2}{\gamma}\left(\frac{c}{\alpha}\right)^{2},
$$


where $P_{m}$ denotes the magnetic pressure. A low- $\beta$ plasma, such as the corona, is thus one for which the Alfven speed greatly exeeds the sound speed, $\alpha>c$. Wave propagation, then, involves the two speeds $c$ and $\alpha$. In fact, the sound speed exists and inter into a description of propagation speeds only in the combination with Alfven speed. As a result, the wave speed $W_{s}$ is such, $S_{s}<W_{s}<F_{s}$, where $F_{s}$ and $S_{s}$ are the fast and slow speeds and defined by

$$
F_{s}^{2}=c^{2}+\alpha^{2}, \quad S_{s}^{2}=c^{-2}+\alpha^{-2}
$$

Moreover, $F_{s}$ will be refered to as magnetoacoustic speed; it is super-sonic and super-Alfvenic. By contrast, $S_{s}$ is both sub-sonic and sub-Alfvénic.

It is easy to see that the maximum of the kinetic energy $\operatorname{Max}(K) \propto\left(\frac{1}{|1+R|}\right)$. As a result, when the reflection coefficient $R \rightarrow-1$, we have $\operatorname{Max}(K) \rightarrow \infty$. This occures when

$$
\theta+2 r \log x_{0} \rightarrow \pm(2 n+1) \pi .
$$

We call this equation, resonence equation.

From the above discussion and the asymptotic behavior of the solution, expressed in (4.9), we have the following observations:

(A) It is clear that $|R|=1$. As a result, the magnetic field produces a nonabsorbing critical layer, below it the solution can be written as a linear combination of an upward and a downward propagating wave with the same wavelengths. Above the critical layer the solution decays exponentially with altitude. Thus, the critical layer separates two distinct regions with different physical properties.

(B) In the critical layer the reflection and the wave modification take place. Since $|R|=1$, the tunneling is very weak while the reflection is very strong. In this case the total energy of the wave is divided equally between the incident and reflected waves.

(C) Since the Magnetoatmosphere is ideal, there is a physical mechanism for dissipation. Thus, we expect the motion to continue in this form and become one of the main sources of energy in the solar atmosphere.

(D) As a result of (B) and (C) we see that the heating process is an acoustic one below the critical layer because the compressibility force dominates the oscillatory process. As we move from a region of weak magnetic field to another one with a strong magnetic field, the heating process becomes magnetoacoustic.

\section{References}

[1] ALKAHBY, H., "Reflection and dissipation of hydromagnetic waves in a viscous and thermally conducting isothermal atmosphere," Geophys.Astroph.Fluid.Dynam., 72, (1993), 197-207.

[2] ALKAHBY, H., "The dissipation of magneto-acoustic waves," Computer Math. Applic., Vol. 27, (1994), 9-15.

[3] ALKAHBY, H., and YANOWITCH, M., "Reflection of vertically propagating waves in a thermall conducting isothermal atmosphere with a horizontal magnetic field," Geophys. Astroph. Fluid. Dynam. Vol. 56, (1991), 227-235. 


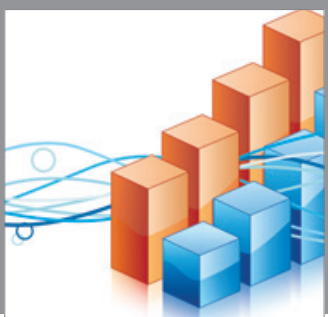

Advances in

Operations Research

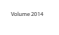

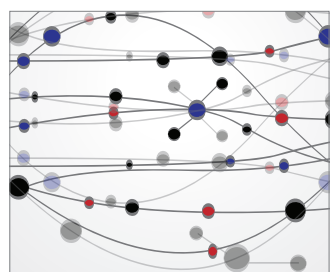

\section{The Scientific} World Journal
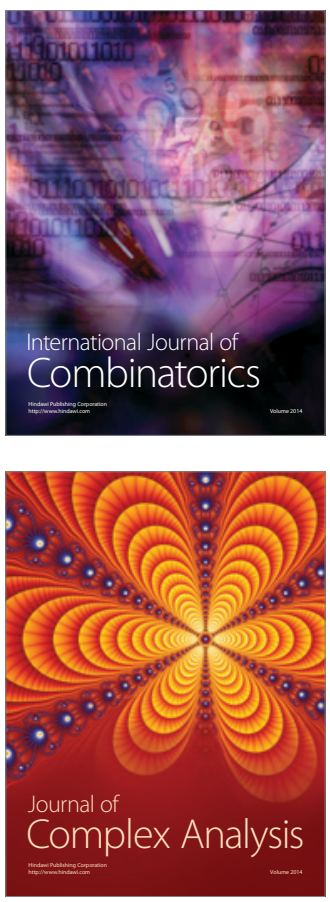

International Journal of

Mathematics and

Mathematical

Sciences
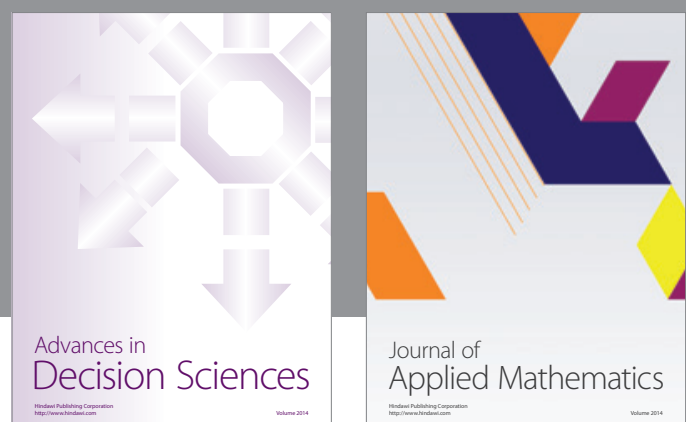

Journal of

Applied Mathematics
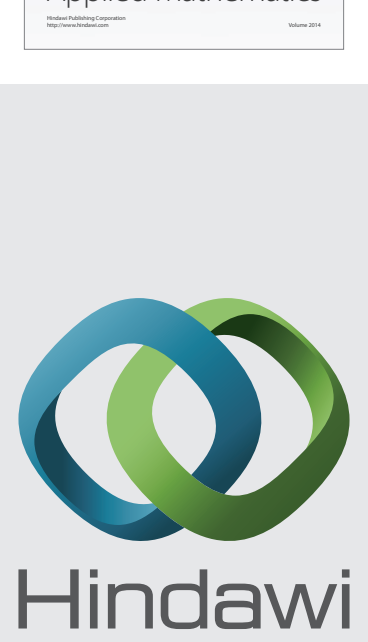

Submit your manuscripts at http://www.hindawi.com
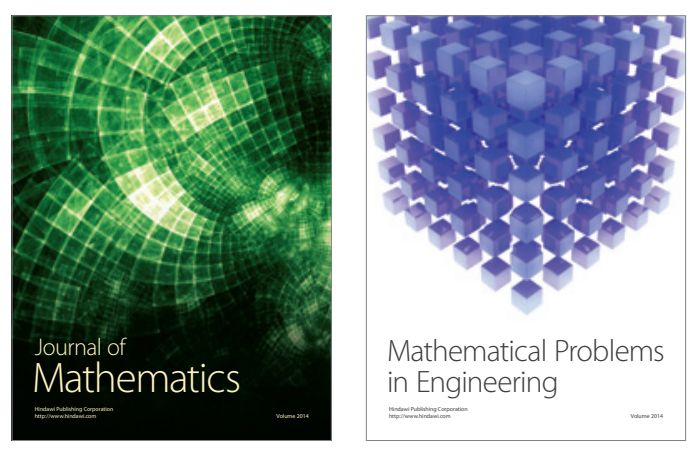

Mathematical Problems in Engineering
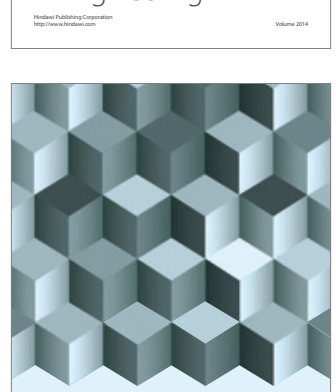

Journal of

Function Spaces
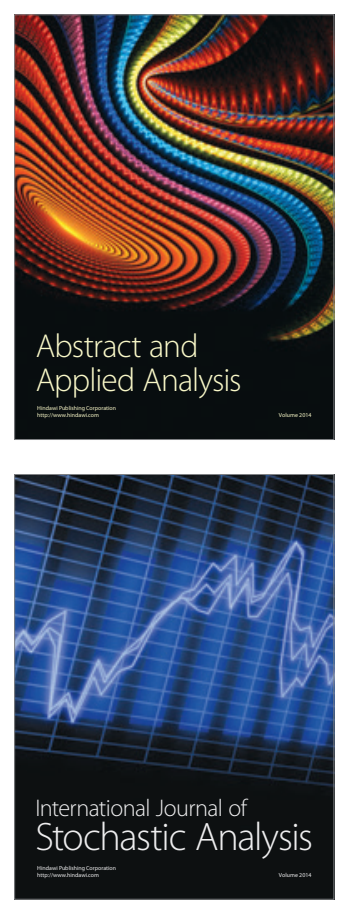

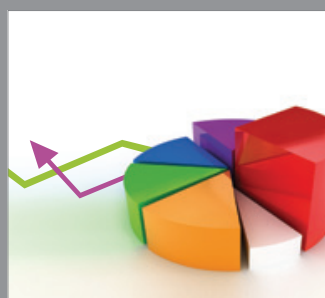

ournal of

Probability and Statistics

Promensencen
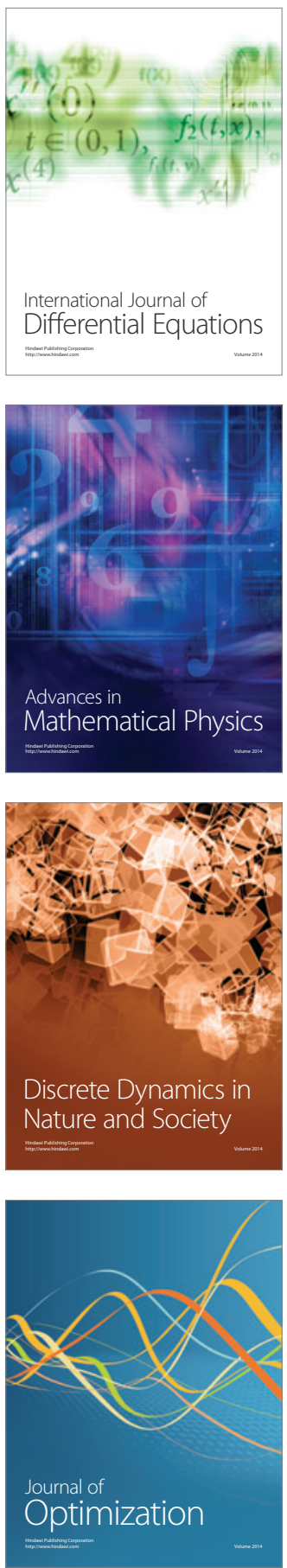